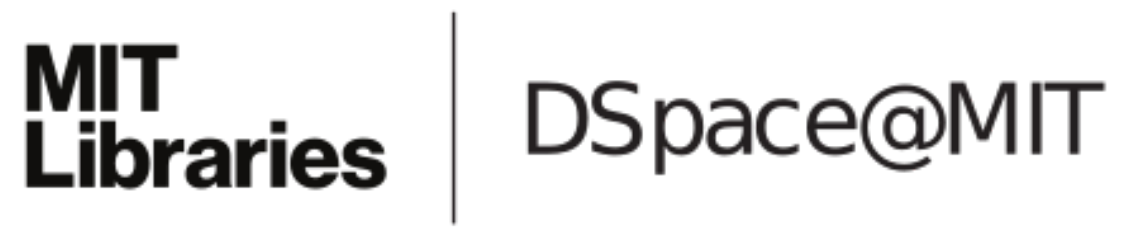

MIT Open Access Articles

Pulling bubbles from a bath

The MIT Faculty has made this article openly available. Please share how this access benefits you. Your story matters.

Citation: Kao, Justin C. T., Andrea L. Blakemore, and A. E. Hosoi. "Pulling Bubbles from a Bath." Physics of Fluids 22.6 (2010): 061705.

As Published: http://dx.doi.org/10.1063/1.3455107

Publisher: American Institute of Physics (AIP)

Persistent URL: http://hdl.handle.net/1721.1/78569

Version: Final published version: final published article, as it appeared in a journal, conference proceedings, or other formally published context

Terms of Use: Article is made available in accordance with the publisher's policy and may be subject to US copyright law. Please refer to the publisher's site for terms of use. 


\title{
Pulling bubbles from a bath
}

\author{
Justin C. T. Kao, ${ }^{\text {a) }}$ Andrea L. Blakemore, and A. E. Hosoi \\ Department of Mechanical Engineering, Massachusetts Institute of Technology, Cambridge, \\ Massachusetts 02139, USA
}

(Received 10 March 2010; accepted 26 May 2010; published online 18 June 2010)

\begin{abstract}
Deposition of bubbles on a wall withdrawn from a liquid bath is a phenomenon observed in many everyday situations - the foam lacing left behind in an emptied glass of beer, for instance. It is also of importance to the many industrial processes where uniformity of coating is desirable. We report work on an idealized version of this situation, the drag-out of a single bubble in Landau-LevichDerjaguin flow. We find that a well-defined critical wall speed exists, separating the two regimes of bubble persistence at the meniscus and bubble deposition on the moving wall. Experiments show that this transition occurs at $\mathrm{Ca}^{*} \sim \mathrm{Bo}^{0.73}$. A similar result is obtained theoretically by balancing viscous stresses and gravity. (C) 2010 American Institute of Physics. [doi:10.1063/1.3455107]
\end{abstract}

Many studies of liquid drag-out by a moving plate are motivated by the need to understand and control various industrial coating processes, particularly dip coating, the simplest and most fundamental method of covering objects with thin layers of liquid. ${ }^{1,2}$ In dip coating, an object is submerged in an open bath. When it is withdrawn, the no-slip condition leads to the deposition of a thin film on its surface. This flow, reduced to its essentials - a wetting solid wall, a meniscus, and the relative motion between the two-is found in a variety of situations, from the coating of plates and fibers, to the deposition of liquid on the walls of a draining vessel, to the motion of bubbles through tubes. Typical quantities of interest are the deposited film thickness and fluid flow rate, and their dependence on the system parameters.

In early mathematical analyses, Landau and Levich ${ }^{3}$ and Derjaguin $^{4,5}$ found that the film thickness $h$ scales like $h \sim \ell_{c} \mathrm{Ca}^{n}$, where $1 / \ell_{c} \equiv \sqrt{\rho g / \sigma}$ is the curvature of the meniscus, $\mathrm{Ca} \equiv \mu U / \sigma$ is the capillary number, and $U$ is the wall speed. The exponent is $n=\frac{2}{3}$ for $\mathrm{Ca} \ll 1$ and $n=\frac{1}{2}$ for $\mathrm{Ca} \gg 1$. Since that time, researchers have expanded our understanding to include higher-order asymptotics, ${ }^{6}$ nonuniqueness of solutions, ${ }^{7}$ non-Newtonian liquids, ${ }^{8}$ inertial effects, ${ }^{9,10}$ gravitational drainage, ${ }^{11,12}$ fiber and tube ${ }^{13-17}$ geometries, and surfactants. ${ }^{18}$ Investigators have also considered other aspects of dip coating, such as wetting failure ${ }^{19,20}$ and air entrainment, ${ }^{21-25}$ both as model systems in dynamic wetting, and because air entrainment may lead to coating defects.

Yet, coating defects due to air entrainment depend not only on the creation of bubbles but also on their subsequent effect on the liquid film. It is well-known that bubbles floating on a free surface experience an attraction (the "Cheerios effect" ${ }^{\text {"26 }}$ ) to wetting menisci such as those created by a partially submerged object. Through this aggregation at menisci, bubbles can alter coating thickness and uniformity, both by altering the effective meniscus curvature, and in the deposition of bubbles on the object through drag-out. Curiously, to our knowledge these aspects of dip coating have received little scientific attention, despite their manifestation in situa-

a)Electronic mail: kaoj@mit.edu. tions as common as the foam lacing left behind in empty beer glasses. Of course, the phenomenon of bubble drag-out is not limited to beer-the authors have observed it in many situations including glasses of milk (Fig. 1), cups of coffee, chocolate-coated strawberries, and less deliciously, in laboratory bottle-roller mixing devices-and it is clearly pertinent to the many industrial processes involving LandauLevich-Derjaguin type flows.

In this letter, we report work on the drag-out of a single isolated bubble initially situated at the meniscus of a Landau-Levich-Derjaguin flow. We observe a well-defined critical wall speed. Below the critical speed, the bubble remains at the meniscus as the wall is withdrawn. However, if the wall speed is increased above critical, bubble behavior undergoes a sharp transition as this stable equilibrium vanishes; the bubble then experiences rapid drag-out and deposition. Our aim is to investigate the dependence of this transition on fluid properties and wall motion.

A diagram of the problem is shown in Fig. 2(a). We consider a vertical wall withdrawn from the bath at a rate $U$, with a bubble of characteristic width $w$ sitting at the corner where the wall exits the bath. We consider a Newtonian fluid with viscosity $\mu$ and constant surface tension $\sigma$. The thickness of liquid deposited on the wall is $h$, and $\ell_{P}$ and $\ell_{c}$ are the characteristic sizes of the Plateau border and meniscus, respectively. If we take these three length scales to be functions of the flow, six independent dimensional quantities are left, yielding three nondimensional groups, the Reynolds number, the capillary number, and the Bond number

$$
\operatorname{Re} \equiv \frac{\rho U \ell_{c}}{\mu}, \quad \mathrm{Ca} \equiv \frac{\mu U}{\sigma}, \quad \text { Bo } \equiv \frac{\rho g w^{2}}{\sigma} .
$$

In the limit that inertia is negligible $(\operatorname{Re} \ll 1)$, the flow can be expressed as a function of capillary and Bond numbers only, $F(\mathrm{Ca}, \mathrm{Bo})=0$. Thus we expect the critical capillary number for bubble drag-out, $\mathrm{Ca}^{*}$, to depend only on Bond number, $\mathrm{Ca}^{*}=\mathrm{Ca}^{*}(\mathrm{Bo})$.

To estimate this dependence, we consider forces acting on the bubble while it remains stationary in the laboratory frame. First, an upward force is exerted on the bubble 


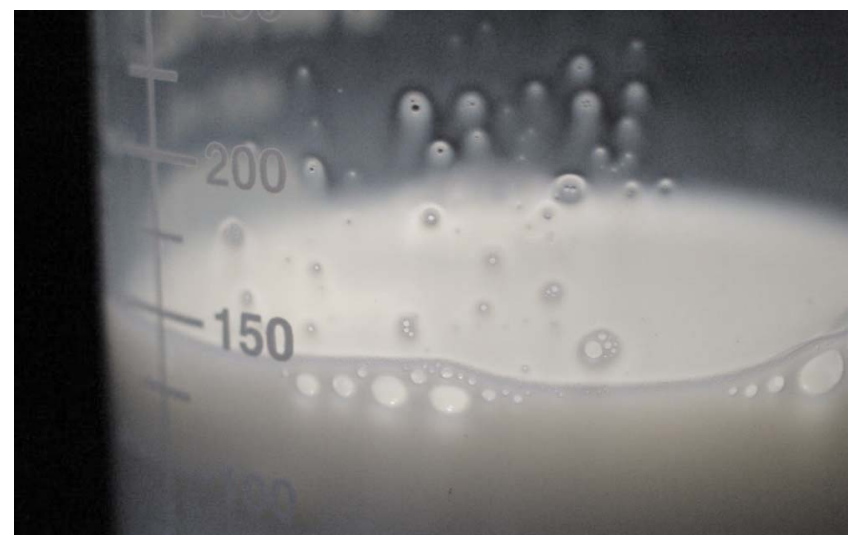

FIG. 1. (Color online) Bubble deposition in a half-emptied beaker of milk. Smaller bubbles are readily deposited on the sides of the beaker by a retreating liquid level, while larger bubbles tend to remain at the meniscus.

through viscous drag against the moving wall. This is analogous to the pressure drop needed to move a train of bubbles through a capillary, or the wall friction experienced by plug flow of foams. Therefore, we may neglect the contribution of the thin films and approximate the viscous drag by forces on the Plateau border only, ${ }^{27}$ which in turn are proportional to the projected length of the Plateau border in the direction of wall motion. ${ }^{28}$ For bubbles in capillaries, Bretherton ${ }^{16}$ found $F_{\mu} \propto \mathrm{Ca}^{2 / 3}$. Strictly, this result is only valid for $\mathrm{Ca}$ considerably smaller than unity, whereas in our case $\mathrm{Ca}^{*} \sim \mathcal{O}(1)$. However, the error here is unavoidable; ascribing the drag force to a Bretherton-type flow is already a significant approximation to the complex geometry of this problem. It would be equally valid to take the viscous drag for wall slip of foams, but numerical results in that case ${ }^{29}$ disagree with those for bubbles in capillaries ${ }^{30}$ with at least as much discrepancy as the error incurred by using Bretherton's relationship outside its range of validity. Finally, we note that analytical results for our geometry appear to be infeasible due to the substantial deformation of the interfaces-indeed researchers have needed to resort to numerical computation for $\mathrm{Ca} \sim \mathcal{O}(1)$ behavior of both Bretherton-type flows as well as wall slip of foams. Therefore we proceed with the only available scaling, namely Bretherton's, and obtain $F_{\mu} \sim \mathrm{Ca}^{2 / 3} \sigma w$ while acknowledging its limitations.

In steady state, the drag force must be balanced by either surface tension or gravity, or both. The surface tension force is the derivative of the extra surface area created by bubble deformation. Modeling the bubble as a prolate spheroid with surface area $A_{\sigma}$, this is $F_{\sigma} \sim \sigma\left(\mathrm{d} A_{\sigma} / \mathrm{d} a\right)$, where $2 a$ is the major axis and the derivative is taken with fixed volume. We introduce the parameter $\delta$ such that $a=a_{0}(1+\delta)$, where $a_{0} \approx w / 2$ is the sphere-equivalent radius of the bubble, and find that $F_{\sigma} \sim \pi \sigma a_{0} f(\delta)$, where $f(\delta)$ has a maximum of $\approx 2.78$ at $\delta \approx 1.19$. Ignoring constant factors, this gives $F_{\sigma} \lesssim \sigma w$.

Finally, we approximate the gravitational force as the weight of liquid beneath the bubble, which is lifted by some small distance $\Delta z \lesssim \ell_{c}$ as the bubble rises. This weight is $F_{g} \lesssim \rho g \ell_{c} w^{2}$.

We do not know a priori whether $F_{\mu} \sim F_{\sigma}$ or $F_{\mu} \sim F_{g}$,

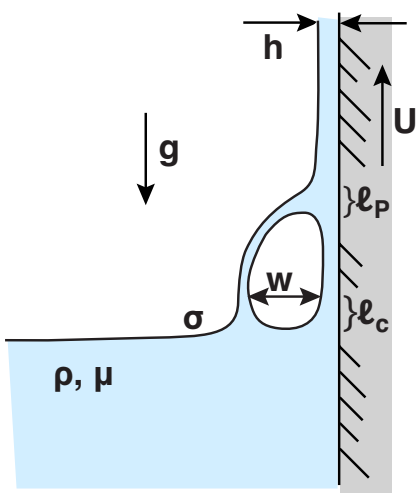

(a)

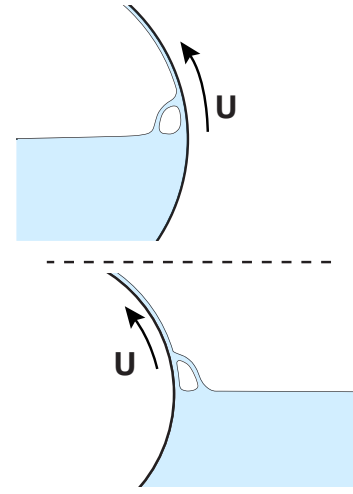

(b)
FIG. 2. (Color online) Schematic diagram of bubble drag-out. (a) A wall is withdrawn from a bath while a bubble remains at the meniscus. (b) Experiments were conducted on the interior or exterior surfaces of rotating cylinders.

but experiments (described below) show that the critical wall speed $U^{*}$ increases with bubble size $w$. A balance of viscous drag with gravity is consistent with this observation, whereas viscous drag balanced by surface tension cannot reproduce this behavior even qualitatively. Therefore we conclude that viscous drag and gravity are the dominant forces, ${ }^{31}$ obtaining the prediction that the critical wall speed is defined by

$$
\mathrm{Ca}^{*} \sim \mathrm{Bo}^{3 / 4}
$$

or, equivalently, $U^{*} \sim \sigma^{1 / 4}(\rho g)^{3 / 4} w^{3 / 2} / \mu$.

To test this prediction, we measured the critical wall speed experimentally. In order to obtain a steady state, we used a rotating cylinder to approximate the continuously moving flat wall [Fig. 2(b)], which carries the unfortunate side effect of introducing two additional parameters, wall curvature $\kappa$ and wall-fluid angle $\theta$. The fluid level was controlled such that $\theta \approx 90^{\circ}$ (equivalent to the flat wall geometry), while $\kappa$ was varied by conducting experiments on both the interiors and exteriors of cylinders, and by using cylinders ranging in diameter from 2.5 to $10.0 \mathrm{~cm}$. Isolated bubbles were introduced at the meniscus while the wall was stationary, and their widths $w$ were measured photogrammetrically using a Canon EOS 10D camera with $100 \mathrm{~mm}$ macrolens. [Contrary to the simplified depiction in Fig. 2(a), $w$ was measured in the horizontal direction parallel to the wall, i.e., into the page.] Subsequently, wall speed was slowly increased until the bubble was dragged out. This speed, as calculated from an encoder on the drive motor, was taken to be the critical wall speed. Photographs of a bubble against a stationary wall and against a wall moving near the critical speed are shown in Fig. 3.

Two fluid types were used, mixtures of Lyle's golden syrup with water (henceforth referred to as "Lyle's") and mixtures of water with polyvinylpyrrolidone ("PVP K60"). Temperature- and composition-dependent viscosities were measured $^{32}$ to range from $0.29 \mathrm{~Pa}$ s for dilute PVP K60 solution to 8.5 $\mathrm{Pa}$ s for pure Lyle's syrup. These tabulated data were combined with infrared thermometer readings to correct for temperature variations during the experiments. Surface tensions at room temperature $\left(23 \pm 2{ }^{\circ} \mathrm{C}\right)$ were found ${ }^{33}$ 

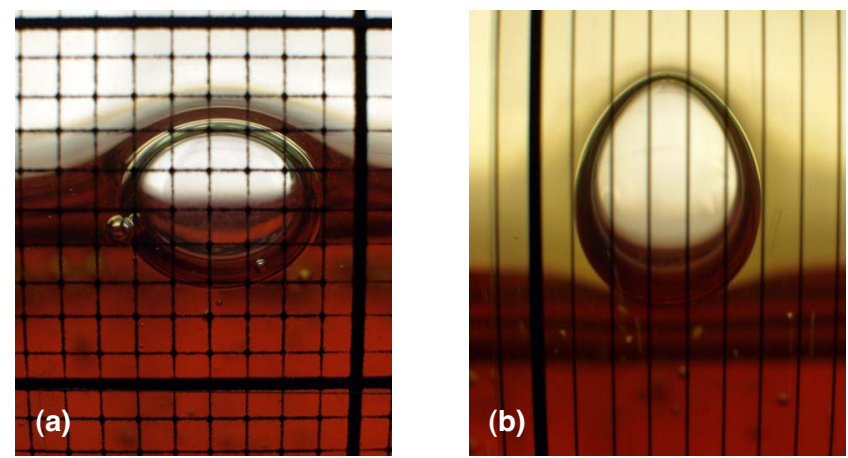

FIG. 3. (Color online) Stable bubbles in Lyle's-water mixture inside a cylinder. The grid lines are spaced at $1 \mathrm{~mm}$ and the view is through the cylinder wall. (a) Bubble with stationary wall. (b) Bubble with moving wall near the critical speed. Significant deformation occurs, particularly for larger bubbles.

to be $0.076 \mathrm{~N} / \mathrm{m}$ for Lyle's and $0.067 \mathrm{~N} / \mathrm{m}$ for PVP K60. Wettability of the cylinders was observed to be a major factor in the reproducibility of the critical wall speed determination. Since we did not wish to introduce surfactants into the system, cylinders were constrained to be either glass or the original Lyle's bottles (which appear to have adsorbed syrup molecules over time). An additional constraint on our setup was the stability of the bubbles; for instance, silicone oil of similar viscosities was unable to produce long-lasting bubbles. $^{34}$

Representative data are shown in Fig. 4(a) for a single value of wall curvature. Each point corresponds to the measured width and critical wall speed of a different bubble. Data from both fluid types and five different fluid viscosities are shown. When nondimensionalized and plotted on logarithmic axes, it is seen that the data collapse onto a single trend [Fig. 4(b)], reflecting that a power law $\mathrm{Ca}^{*} \sim \mathrm{Bo}^{\alpha}$ is consistent with our data.

When we vary both fluid composition and wall curvature, and plot the best-fit ${ }^{35}$ power law exponents versus fluid viscosity for all of our experimental runs, we obtain Fig. 5(a). Here each point is the exponent extracted from a run of data collected in a single sitting, and symbols and shades correspond to wall curvature. There is a slight viscosity dependence for fluid on the interior of smaller diameter cylin-
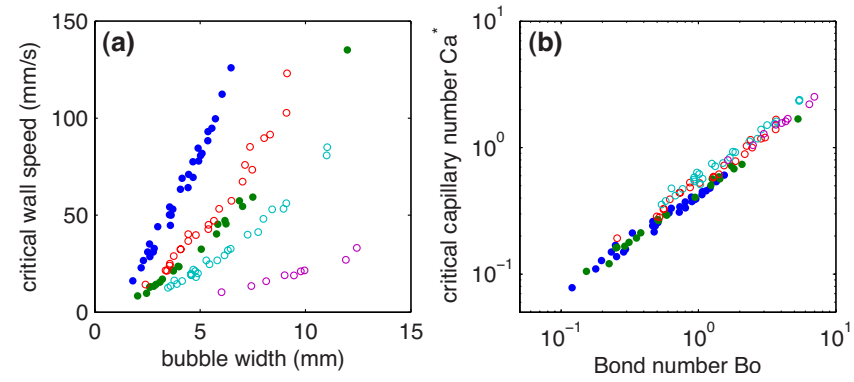

FIG. 4. (Color online) Data taken for bubbles outside a $10.0 \mathrm{~cm}$ cylinder for five different fluid viscosities. Each point corresponds to the width and critical wall speed measured for an individual bubble. Filled circles $(\bullet)$ are PVP K60 and open circles $(\bigcirc)$ are Lyle's. Viscosity varies from 0.32 to 6.0 Pa s. (a) As measured. (b) Nondimensionalized and plotted on logarithmic axes.
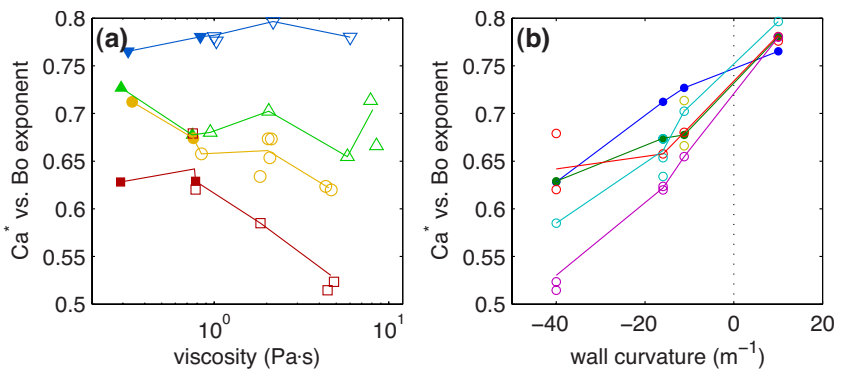

FIG. 5. (Color online) (a) Best-fit exponents plotted vs fluid viscosity. Filled symbols are PVP K60 runs and open symbols are Lyle's runs. Wall curvatures are $40 \mathrm{~m}^{-1}(\square), 16 \mathrm{~m}^{-1}(\bigcirc)$, and $11 \mathrm{~m}^{-1}(\triangle)$ for interior geometry, and $10 \mathrm{~m}^{-1}(\nabla)$ for exterior geometry. Solid lines show best-fit slopes calculated by combining all data points for each particular fluid mixture. (b) Curvature dependence of the exponent, plotted for each fluid mixture used. Filled circles $(\bullet)$ are PVP K60 and open circles $(\bigcirc)$ are Lyle's. Shades correspond to different mixture compositions.

ders $(\square)$, likely due to changes in the wall-fluid angle at higher rotation rates. However, this dependence diminishes with increasing radius of curvature and is absent altogether for fluid on the exterior of a rotating cylinder ( $\nabla$ and Fig. 4), suggesting that our nondimensionalization has indeed correctly accounted for fluid viscosity.

We explore the curvature dependence by plotting the power law exponents as a function of wall curvature [Fig. 5(b)]. A clear trend is seen. Interpolating these data suggests that for a flat wall, the relationship

$$
\mathrm{Ca}^{*} \sim \mathrm{Bo}^{0.73},
$$

holds, which is in good agreement with our theoretical prediction (1).

Finally, the complete data set is shown in Fig. 6. It can be seen that the effect of wall curvature is greatest at high wall speeds and large bubble sizes, as is expected.

In this initial investigation of bubble deposition, we have necessarily neglected a number of interesting and important

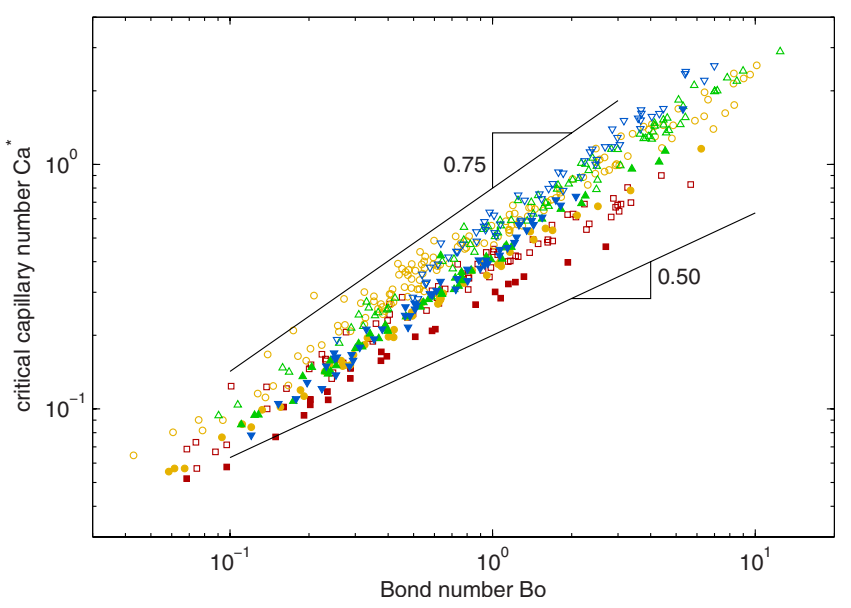

FIG. 6. (Color online) Complete data set. Each point represents a bubble width and critical wall speed measurement. Symbol shade and shape denote wall curvature as in Fig. 5(a) $(\square \bigcirc \Delta \nabla)$ from concave to convex. Filled symbols are for PVP K60 and open symbols are for Lyle's. The larger scatter at high $\mathrm{Bo}$ and $\mathrm{Ca}^{*}$ reflects the greater curvature dependence under these conditions. 
aspects of this problem. Among these are the interaction of multiple bubbles (as in foams and froth), the influence of surfactants (known to be significant for coating flows), and non-Newtonian effects. In addition, we have considered only the initial drag-out of bubbles; the thinning of the liquid coating above bubbles that remain at the meniscus, as well as the subsequent evolution of bubbles after deposition, including effects such as capillary suction of the coating film and gravitational drainage of bubble Plateau borders, remain as open problems. The latter two effects are clearly observed in the photograph of Fig. 1.

In summary, we have investigated the phenomenon of bubble drag-out and deposition in the Landau-LevichDerjaguin flow. Using dimensional analysis, we have shown that for a flat wall, the critical wall speed for drag-out must be of the form $\mathrm{Ca}^{*}=\mathrm{Ca}^{*}(\mathrm{Bo})$. A simple force balance between viscous drag and the weight of liquid beneath a bubble gave us $\mathrm{Ca}^{*} \sim \mathrm{Bo}^{3 / 4}$, while experiments suggest a relationship of $\mathrm{Ca}^{*} \sim \mathrm{Bo}^{0.73}$. It is seen that the data is wellapproximated by a power-law relationship over an order-ofmagnitude parameter range, both in fluid viscosity and in bubble width.

J.C.T.K. was supported by an NSF Mathematical Sciences Postdoctoral Research Fellowship, Contract No. DMS0803083. A.L.B. was funded by the MIT Undergraduate Research Opportunities Program. Additional funding was also provided by NSF Contract No. CTS-0624830.

${ }^{1}$ S. F. Kistler and P. M. Schweizer, Liquid Film Coating (Chapman and Hall, London, 1997).

${ }^{2}$ S. J. Weinstein and K. J. Ruschak, "Coating flows," Annu. Rev. Fluid Mech. 36, 29 (2004).

${ }^{3}$ L. Landau and B. Levich, "Dragging of a liquid by a moving plate," Acta Physicochim. URSS 17, 42 (1942).

${ }^{4}$ B. Derjaguin, "Thickness of liquid layer adhering to walls of vessels on their emptying and the theory of photo- and motion picture film coating," C. R. (Dokl.) Acad. Sci. URSS 39, 13 (1943).

${ }^{5} \mathrm{~B}$. Derjaguin, "On the thickness of the liquid film adhering to the walls of a vessel after emptying," Acta Physicochim. URSS 20, 349 (1945).

${ }^{6}$ S. D. R. Wilson, "The drag-out problem in film coating theory," J. Eng. Math. 16, 209 (1982).

${ }^{7}$ J. H. Snoeijer, A. J. Ziegler, B. Andreotti, M. Fermigier, and J. Eggers, "Thick films of viscous fluid coating a plate withdrawn from a liquid reservoir," Phys. Rev. Lett. 100, 244502 (2008).

${ }^{8}$ J. S. Ro and G. M. Homsy, "Viscoelastic free surface flows: Thin film hydrodynamics of Hele-Shaw and dip coating flows," J. Non-Newtonian Fluid Mech. 57, 203 (1995).

9 J. P. Kizito, Y. Kamotani, and S. Ostrach, "Experimental free coating flows at high capillary and Reynolds number," Exp. Fluids 27, 235 (1999).

${ }^{10}$ B. Jin, A. Acrivos, and A. Münch, "The drag-out problem in film coating," Phys. Fluids 17, 103603 (2005).

${ }^{11}$ D. A. White and J. A. Tallmadge, "Theory of drag out of liquids on flat plates," Chem. Eng. Sci. 20, 33 (1965).

${ }^{12}$ R. P. Spiers, C. V. Subbaraman, and W. L. Wilkinson, "Free coating of a Newtonian liquid onto a vertical surface," Chem. Eng. Sci. 29, 389 (1974).
${ }^{13}$ D. A. White and J. A. Tallmadge, "A gravity corrected theory for cylinder withdrawal," AIChE J. 13, 745 (1967).

${ }^{14}$ S. D. R. Wilson, "Coating flow on to rods and wires," AIChE J. 34, 1732 (1988).

${ }^{15}$ D. Quéré, "Fluid coating on a fiber," Annu. Rev. Fluid Mech. 31, 347 (1999).

${ }^{16}$ F. P. Bretherton, "The motion of long bubbles in tubes," J. Fluid Mech. 10, 166 (1961).

${ }^{17}$ P. Aussillous and D. Quéré, "Quick deposition of a fluid on the wall of a tube," Phys. Fluids 12, 2367 (2000).

${ }^{18}$ R. Krechetnikov and G. M. Homsy, "Surfactant effects in the LandauLevich problem," J. Fluid Mech. 559, 429 (2006).

${ }^{19}$ J. Eggers and H. A. Stone, "Characteristic lengths at moving contact lines for a perfectly wetting fluid: The influence of speed on the dynamic contact angle," J. Fluid Mech. 505, 309 (2004).

${ }^{20}$ M. Maleki, E. Reyssat, and D. Quéré, "On the Landau-Levich transition," Langmuir 23, 10116 (2007).

${ }^{21}$ S. D. R. Wilson and A. F. Jones, "The entry of a falling film into a pool and the air-entrainment problem,” J. Fluid Mech. 128, 219 (1983).

${ }^{22}$ M. N. Esmail and M. T. Ghannam, "Air entrainment and dynamic contact angles in hydrodynamics of liquid coating," Can. J. Chem. Eng. 68, 197 (1990).

${ }^{23}$ O. Cohu and H. Benkreira, "Air entrainment in angled dip coating," Chem. Eng. Sci. 53, 533 (1998).

${ }^{24}$ J. Eggers, "Air entrainment through free-surface cusps," Phys. Rev. Lett. 86, 4290 (2001).

${ }^{25}$ P. G. Simpkins and V. J. Kuck, "On air entrainment in coatings," J. Colloid Interface Sci. 263, 562 (2003).

${ }^{26}$ D. Vella and L. Mahadevan, "The Cheerios effect," Am. J. Phys. 73, 817 (2005).

${ }^{27}$ J. Ratulowski and H.-C. Chang, "Transport of gas bubbles in capillaries," Phys. Fluids A 1, 1642 (1989).

${ }^{28}$ I. Cantat, N. Kern, and R. Delannay, "Dissipation in foam flowing through narrow channels," Europhys. Lett. 65, 726 (2004).

${ }^{29}$ A. Saugey, W. Drenckhan, and D. Weaire, "Wall slip of bubbles in foams," Phys. Fluids 18, 053101 (2006).

${ }^{30}$ M. D. Giavedoni and F. A. Saita, "The axisymmetric and plane cases of a gas phase steadily displacing a Newtonian liquid-A simultaneous solution of the governing equations," Phys. Fluids 9, 2420 (1997).

${ }^{31}$ Surface tension is not negligible however. It acts to couple the viscous drag experienced at the top of the bubble to the weight of liquid beneath the bubble, and it is also embedded in the Bretherton scaling due to interfacial deformation.

${ }^{32}$ Viscosity measurements were conducted using a TA Instruments AR-G2 rheometer with $60 \mathrm{~mm}$ cone and Peltier plate. The measured viscosities were constant to within $6 \%$ over a range of shear rates $\dot{\gamma} \in[0.1,10] \mathrm{s}^{-1}$, with the exception of the least viscous Lyle's-water mixture, which had a variation of $15 \%$.

${ }^{33}$ Surface tension measurements were made using a Krüss K10ST with platinum Wilhelmy plate. Results were largely independent of mixture composition in the range of compositions we used. We did not correct for temperature dependence of the surface tension.

${ }^{34}$ We have verified that the addition of the surfactant polysorbate 20 to the PVP K60 mixture does indeed lower the critical wall speed for bubble drag-out. This, along with the relatively large surface tensions measured, strongly suggests that bubble longevity in our two mixtures is due to favorable intermolecular potentials in the free film rather than the presence of surfactants.

${ }^{35}$ Because both the abscissa and ordinate may contain errors, we use the least product regression rather than ordinary least squares, yielding a fit line with the desirable properties of being symmetric and scale invariant with respect to both variables (Ref. 36).

${ }^{36} \mathrm{P}$. A. Samuelson, "A note on alternative regressions," Econometrica 10, 80 (1942). 